\title{
Students' Misconceptions about Locating Integers and Decimals on Number Line
}

\author{
Dr. Zarina Akhtar ${ }^{1}$
}

\begin{abstract}
This paper explores misconceptions about locating integers and decimals on horizontal and vertical number lines as revealed by the students' responses enrolled at Federal Government Secondary Schools in Islamabad. The instrument was a test based on 4 parts containing integers and decimals placing on horizontal and vertical number lines. 368 students were the sample of the study. The analysis of students' incorrect responses ranged from $4 \%$ to $62 \%$. The analyses of students' incorrect responses helped for the investigation of students' misconceptions. Four misconceptions leading to incorrect placement of integers and decimals were identified. The opposite sign misconception appeared when students placed positive integers in negative region and negative integers in positive region of number line. The other two misconceptions Translating Positive Interval (TPI) and Translating Negative Interval (TNI) appeared by creating the positive and negative part of the number line which emerged through the negative and positive intervals. The 'decimal-point-ignored' misconception was identified when students ignored decimal point and considered the remaining part as whole numbers on horizontal and vertical number lines. The implication for the students and teachers is that, teaching of integers and decimals should not be confined to conceptual understanding only but also through the visual description on number lines which must be focused during teaching.
\end{abstract}

Keywords: Misconceptions, negative integers, positive integers, decimals, horizontal number line, vertical number line.

\section{Introduction}

Numbers in mathematics has significant status. Numbers are presented in graphic and geometric forms. Number line is one of the significant forms of representing numbers. The number line is a diagram, which represents the single position and encodes quantitative information by their position on a horizontal or vertical axis (Mackinlay, 1999). The number line is important because of its relative size. It is used for measuring scales and Cartesian Axes (horizontal and vertical) as well as abstract set of real numbers. Number line on one way gives concept of continuity and on other way the concept of linearity. According to

\footnotetext{
${ }^{1}$ Assistant Professor, Department of Education, IIUI, Email: zarina.akhtar@iiu.edu.pk
} 
Diezman \& Lowrie (2006) number line have three potential advantages for students firstly, it accommodates 'mathematical variability' of concepts e.g. a number line is useful in showing the continuity aspect of rational numbers. Secondly it contributes to 'perceptual variability' of a concept e.g. a fraction can be represented by a number line and a pie diagram. Thirdly number line is a tool for representational transfer.

The number line has important place in school curricula. Placing numbers on number line or identifying integers on number line are being studied during early mathematics teaching. Mathematics is either perceived or conceived as difficult subject because of its abstract nature. The visual description of number line justifies to some extent its abstract nature. It gives representation of different concepts regarding numbers, rational numbers, integers, fractions and decimals. The studies are evident of students' problems and misconceptions about placing numbers on number line (Fuadiah, Suryadi \& Turmudi, 2017). Students' problems in solving mathematical concepts are based on their previous experience and concepts (Schindler, Hußmann, Nilsson \& Bakker, 2017; Schindler, Hußmann, 2013). Sometimes incorrect information may also constitute misconceptions to new knowledge.

\subsection{Objectives of the study}

1. To identify students' misconceptions in locating positive and negative integers on number line.

2. To identify students' misconceptions in locating positive and negative decimals with non-zero integer part.

3. To identify students' misconceptions in locating positive and negative decimals with zero integer part on number line.

\section{Review of Literature}

Students' problems about locating numbers, fractions and decimals on number line have been investigated by different researchers. For instance, Michaelidou, Gagatsis \& Pantazi (2004) studied students' performance in two different translation modes. There were three tasks, recognition of decimals, representation of decimals and translation ability from number line to symbolic expression and vice versa. They have investigated twelve years old student's responses and found translation from number line to symbolic expression seems easy as compared to symbolic representation to number line. They reported students have difficulties in representing decimals on number line but don't specify which type of difficulties they have? Hannula (2003) investigated students' responses for locating fraction $3 / 4$ on a number line. $49 \%$ students' response was correct. Only 5\% students located $3 / 4$ incorrectly between zero and 
one. Quarter of students located it between 2.5 and 3.5 and $1 \%$ answers were between 1 and 2.5. According to research the main difficulty for students was to determine what was the 'whole', where from to calculate the fraction? So the whole number such as 4 can be represented with 4 objects i.e. symbolic representation and $3 / 4$ can be represented by three shaded quarters of a rectangle i.e. visual representation, to represent $3 / 4$ on number line require spatial information (related to visual representation where is the point) and symbolic information (where are symbols for other numbers placed?) so to represent $3 / 4$ on number line it is necessary to read the position of other numbers such as 0 and 1 . The number line therefore appears as a different type of model to students (Diezmann \& Lowrie, 2006).

Peled \& Carraher (2008) discussed students' difficulties in understanding negative numbers. They found students have difficulties in understanding both; the meaning of negative numbers and the operation associated to it. The same was explained by Steinle and Stacey (2004) as reciprocal thinking through the mirror metaphors. They elaborated the reciprocal thinking makes an analogy with the fact that $1 / 73<1 / 6$ and concludes 0.73 is smaller than 0.6 . One other source of difficulty dealing with negative numbers explained by Peled and Carraher (2008) that students are required to understand the order relation and magnitude of numbers at the same time. According to Steinle (2004) certain key concepts in the curriculum, students transform in an active way during teaching these often lead to misconceptions. This is further described by Mclntosh, Stacey, Tromp \& Lightfoot (2000) during the trial of the computer game (flying photographer) for the support of learning size of the decimals. Students developed four strategies at their own which help them place the number on number line. Basically these strategies don't help them in understanding the concept or place value of a number used for placement of specific number but rather it showed associated knowledge for example in section strategies placing all numbers of the form $0.8 \mathrm{xxx}$ in between 0.8 and 0.9 on the basis of the tenth digit. The specific finding of the study was students often succeed on the basis of partial knowledge of how to use number line.

Confrey (1990) cited by Steinle (2004) noted various terms associated with misconceptions such as alternative conceptions, students conceptions, preconceptions, conceptual primitive, private concepts, alternative frame work systematic errors and critical barriers to learning. Different studies have documented various misconceptions about the decimals in sample of school students (Irwin, 1995; Stacy, 2005; Steinle, 2004). Some studies have also been conducted with teachers, and misconceptions and difficulties with decimals have been observed (Putt, 1995; Irwin, 1995; Stacey, Helme, Steinle, Baturo, Irwin \& 
Bana, 2001). The findings showed some teachers share misconceptions in younger students.

Widjaja, Stacey \& Steinle (2011) studied misconceptions of pre-service teachers locating negative decimals on the number line. They found two types of misconceptions; the first is separate negative number ray misconception and second is Translating Positive Interval (TPI) misconceptions. The respondents with TPI misconception know the location of positive and negative numbers correctly on number line. These students know that 1.2 is to the left of 1.3 (on the positive number line) and assume that the same relationship holds for negative numbers so that the -1.2 is to the left of -1.3 . Instead of interpreting -1.2 as (-1$0.2)$ they interpret it as $(-1+0.2)$. Although they neither are unlikely to think in this mathematical way nor would they use the language of translations. The other four variations of TP12, TP13, TP14 and TP15 were also found by placing these same translated positive intervals in slightly different positions. Above defined misconceptions by Steinle, 2004; Steinle and Stacy (2004) and Widjaja, Stacey \& Steinle, (2011) has been the focus of this study. It tried to determine the prevalence of these misconceptions in students' responses about placing integers and decimals on number line.

\subsection{Research Design}

\section{Research Methodology}

The study was descriptive in nature and survey method was used.

\subsection{Population and Sample}

The population of the study was students enrolled at Federal Government Secondary Schools of Islamabad. Federal Directorate of Education was consulted for the administration of tests. The consent of teachers and students were taken. It took 25 to 30 minutes by an average student to complete the test. By cluster sampling technique the whole class was taken as sample. All the students present on data collection day in classes 7, 8 and 9 of 4 schools in Islamabad sector were the sample of the study. 368 students were present on data collection day so these represent the sample of the study.

\subsection{Research Instrument}

The instrument used in this study was self-developed test. The integers $(1,2$, - 1 and - 2) and decimals $(2.1,1.7,2.3,1.8 ;-1.8,-2.3,-1.7,-2.8 ;-0.3,0.2,0.3,-$ $0.2)$ placed on horizontal and vertical number lines were the test. These integers and decimals were previously used by the team of "SMART" (Specific Mathematics Assessments that Reveal Thinking) Project. The integers and decimals for horizontal and vertical number lines were placed on 4 cards (used as test), 2 for horizontal and 2 for vertical number lines. Each card contains 4 
horizontal and 4 vertical number lines. In this way it was 16 number lines on which given integers and decimals have to be placed. Figure 1 shows the test on horizontal number line, which contains negative and positive integers i.e. 2 and 1 , negative and positive decimals with non-zero integer parts i.e. $-1.8,-2.3,2.1$ and 1.7 and positive and negative decimals with zero integer part i.e. -0.3 and 0.2 . The figure 2 shows test on vertical number line, which contains almost similar integers and decimals with little variation. The items on each card are given in Figure 1 and 2.

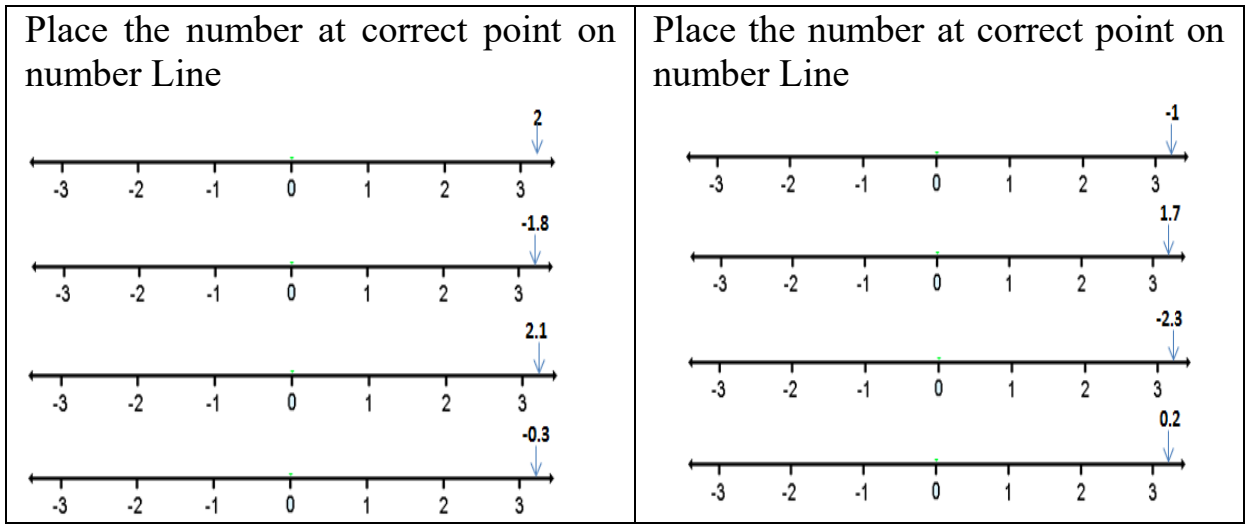

Figure 1: Item 1 and 2 for Horizontal Number Line

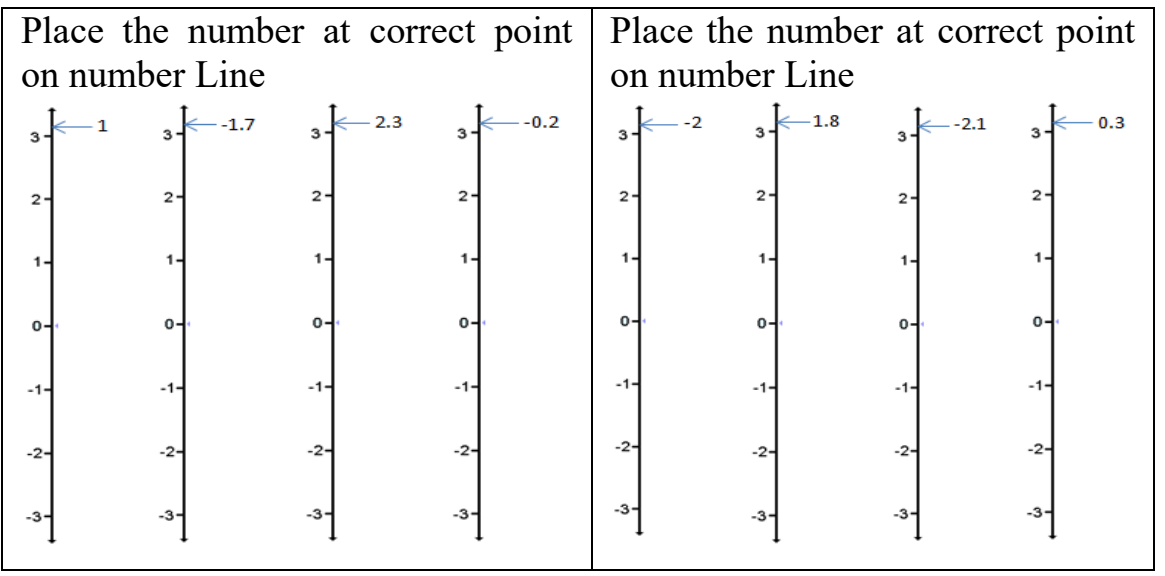

Figure 2: Item 3 and 4 for Vertical Number Line 


\subsection{Data Collection and Data analysis}

Both the cards (instrument) containing number lines were administered to students with the help of their teachers during their class. Students' incorrect responses were analyzed to identify misconceptions about placing numbers on horizontal and vertical number line.

\section{Results}

All the items in Figure 1 and 2 contains 4 questions, based on negative and positive integers, negative and positive decimals with non-zero integer part and zero integer part. The accuracy of students' responses for positive and negative integers is measured as the same number but the accuracy of students' responses for decimal is measured against one tenth above and one tenth below the decimal number for item 1, 2, 3 and 4. The percentage of correct, incorrect and not attempted responses is presented in Fig 3 and 4.

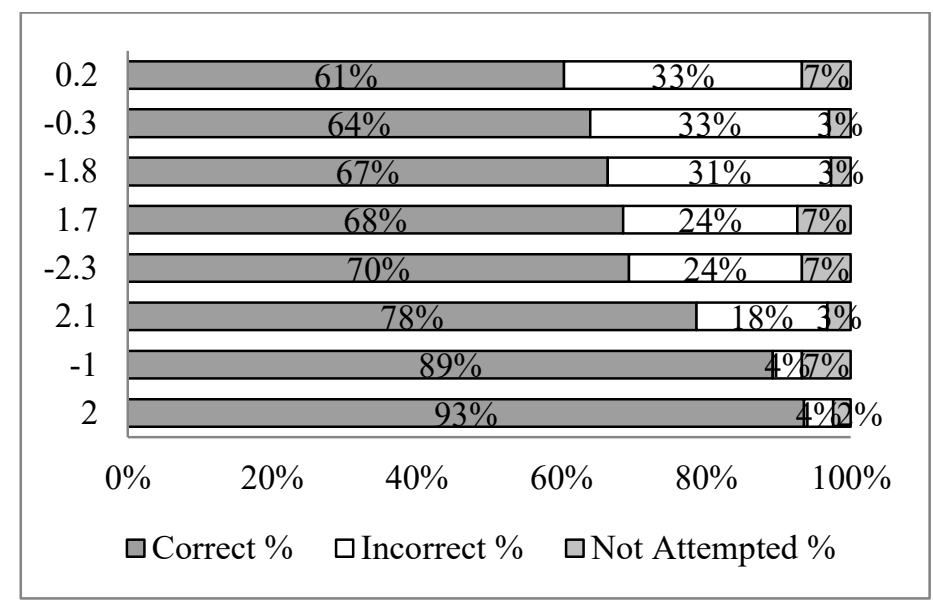

Figure 3: Students' responses on item 1 and 2 on horizontal number line

It seems students have no problem in locating integers on number line as $93 \%$ and $89 \%$ students mark positive and negative integers correctly on number line. Almost 30\% students face problem in locating decimal numbers on number line. 


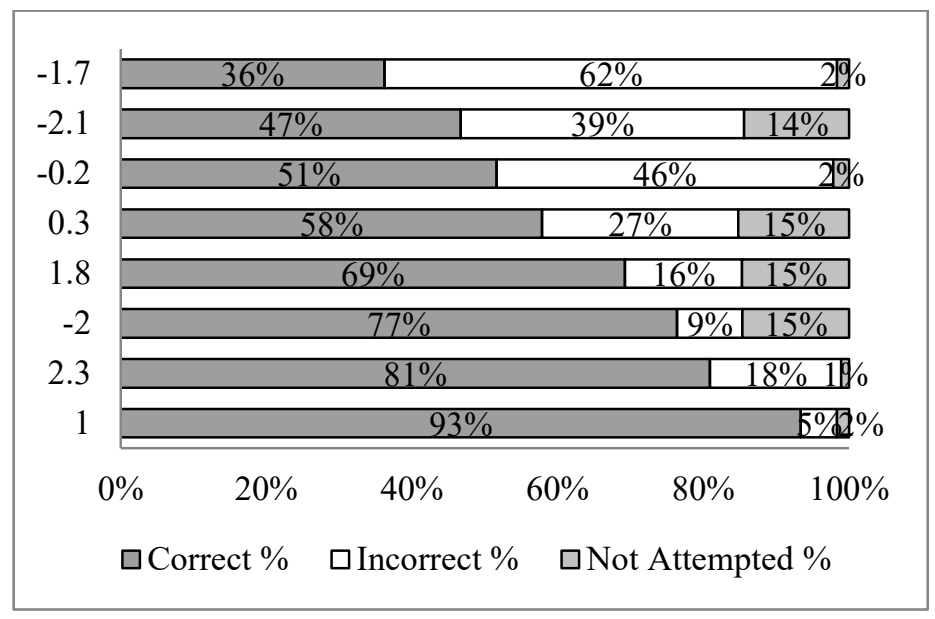

Figure 4: Students' responses on item 3 and 4 on vertical number line

The highest incorrect response is for decimals $(-1.7,-2.1,-0.2)$. Students consistently located decimals at wrong place on number line. The students correct, incorrect and not attempted responses are presented in following tables.

Table 1 Students' responses for placing positive and negative integers on horizontal and vertical number line

\begin{tabular}{ccccccccc}
\hline \multirow{2}{*}{ Number } & \multicolumn{2}{c}{ Correct } & \multicolumn{2}{c}{ Incorrect } & \multicolumn{2}{c}{ Not Attempted } & \multicolumn{2}{c}{ Total } \\
\cline { 2 - 9 } & f & $\%$ & f & $\%$ & f & $\%$ & f & $\%$ \\
\hline 2 & 344 & $93.48 \%$ & 15 & $4.08 \%$ & 9 & $2.45 \%$ & 368 & $100 \%$ \\
1 & 344 & $93.48 \%$ & 18 & $4.89 \%$ & 6 & $1.63 \%$ & 368 & $100 \%$ \\
-1 & 329 & $89.40 \%$ & 14 & $3.80 \%$ & 25 & $6.79 \%$ & 368 & $100 \%$ \\
-2 & 282 & $76.63 \%$ & 32 & $8.70 \%$ & 54 & $14.67 \%$ & 368 & $100 \%$ \\
\hline \multicolumn{2}{c}{$*=$ frequency }
\end{tabular}

Table 2 Students' responses for placing positive decimals numbers on horizontal and vertical number line

\begin{tabular}{ccccccccc}
\hline \multirow{2}{*}{ Number } & \multicolumn{2}{c}{ Correct } & \multicolumn{2}{c}{ Incorrect } & \multicolumn{2}{c}{ Not Attempted } & \multicolumn{2}{c}{ Total } \\
\cline { 2 - 9 } & $\mathrm{f}$ & $\%$ & $\mathrm{f}$ & $\%$ & $\mathrm{f}$ & $\%$ & $\mathrm{f}$ & $\%$ \\
\hline 2.1 & 288 & $78.26 \%$ & 68 & $18.48 \%$ & 12 & $3.26 \%$ & 368 & $100 \%$ \\
1.7 & 251 & $68.21 \%$ & 90 & $24.46 \%$ & 27 & $7.34 \%$ & 368 & $100 \%$ \\
2.3 & 297 & $80.71 \%$ & 67 & $18.21 \%$ & 4 & $1.09 \%$ & 368 & $100 \%$ \\
1.8 & 254 & $69.02 \%$ & 60 & $16.30 \%$ & 54 & $14.67 \%$ & 368 & $100 \%$ \\
\hline
\end{tabular}


Table 3 Students' responses for placing negative decimals with non-zero integer part on horizontal and vertical number line

\begin{tabular}{ccccccccc}
\hline Number & \multicolumn{2}{c}{ Correct } & \multicolumn{2}{c}{ Incorrect } & \multicolumn{2}{c}{ Not Attempted } & \multicolumn{2}{c}{ Total } \\
\cline { 2 - 9 } & $\mathrm{f}$ & $\%$ & $\mathrm{f}$ & $\%$ & $\mathrm{f}$ & $\%$ & $\mathrm{f}$ & $\%$ \\
\hline-1.8 & 245 & $66.58 \%$ & 113 & $30.71 \%$ & 10 & $2.72 \%$ & 368 & $100 \%$ \\
-2.3 & 256 & $69.57 \%$ & 87 & $23.64 \%$ & 25 & $6.79 \%$ & 368 & $100 \%$ \\
-1.7 & 133 & $36.14 \%$ & 229 & $62.23 \%$ & 6 & $1.63 \%$ & 368 & $100 \%$ \\
-2.1 & 172 & $46.74 \%$ & 143 & $38.86 \%$ & 53 & $14.40 \%$ & 368 & $100 \%$ \\
\hline
\end{tabular}

Table 4 Students' responses for placing positive and negative decimals with zero integer part on horizontal and vertical number line

\begin{tabular}{ccccccccc}
\hline \multirow{2}{*}{ Number } & \multicolumn{2}{c}{ Correct } & \multicolumn{2}{c}{ Incorrect } & \multicolumn{2}{c}{ Not Attempted } & \multicolumn{2}{c}{ Total } \\
\cline { 2 - 9 } & $\mathrm{f}$ & $\%$ & $\mathrm{f}$ & $\%$ & $\mathrm{f}$ & $\%$ & $\mathrm{f}$ & $\%$ \\
\hline-0.3 & 235 & $63.86 \%$ & 122 & $33.15 \%$ & 11 & $2.99 \%$ & 368 & $100 \%$ \\
-0.2 & 189 & $51.36 \%$ & 171 & $46.47 \%$ & 8 & $2.17 \%$ & 368 & $100 \%$ \\
0.2 & 223 & $60.60 \%$ & 120 & $32.61 \%$ & 25 & $6.79 \%$ & 368 & $100 \%$ \\
0.3 & 213 & $57.88 \%$ & 99 & $26.90 \%$ & 56 & 0.152174 & 368 & $100 \%$ \\
\hline
\end{tabular}

Although integers seems easy to place on horizontal and vertical number line but for decimals the percentage of incorrect responses are not negligable to ignore.

In following section the students correct and incorrect responses are analysed so that we can evaluate what type of repeated pattrens of responses occurred. These pattrens may be students systematic errors which leads to the misconceptions.

This part of test (instrument) contains items placed on horizontal number line.

\subsection{Placement of $(2$ and -1$)$ negative and positive integers on horizontal number line}

Majority of students marked integers 2 and -1 correctly except 5 and 4 students respectively whose responses are incorrect. These students incorrecly marked 2 at -2 and -1 at 1 . They ignored the sign of the integers. It can be said these students have opposite sign misconception.

\subsection{Placement of $(-1.8$ and -2.3$)$ negative decimals with non-zero integer part on horizontal number line}

The students' responses for locating negative integers on horizontal number line are analyzed. It has been observed that all the incorrect responses were marked at the same point systematically around -1 and -2 from the correct point. Here two types of incorrect responses are observed. For -1.8 some (10) students have added the decimal part in negative integer and place -1.8 at $-0.2(=$ $-1+0.8)$. These students have translated the decimal part to positive side of 
number line. On the other side they (61) have simply ignored the negative sign and place -1.8 at 1.8 on number line. The consistency in this response has been observed in above responses (section 4.1) too. For -2.3 students showed same type of response on both sides of number line towards negative region they (29) have added 0.3 in -2 and place -2.3 at $-1.7(=-2+0.3)$ on positive side they (24) have ignored negative sign and placed -2.3 at 2.3 .

\subsection{Placement of (1.7 and 2.1) positive decimals with non-zero integer part on horizontal number line}

The students correct and incorrect responses for 1.7 and 2.1 been analyzed. They have placed 1.7 at three incorrect places. 8 students placed 1.7 incorrectly at 0.3 they have subtracted 0.7 from 1 and placed it at $0.3(=1-0.7)$. The other (9) students have focused the negative region of number line and they have added 0.7 in -1 and placed at $-0.3(=-1+0.7)$. Third 54 students ignored positive sign and placed 1.7 at -1.7 . They showed opposite sign misconception.

\subsection{Placement of $(-0.3$ and 0.2$)$ negative and positive decimals with zero integer part on horizontal number line}

Students' responses about decimals with zero integer part been analyzed. For - 0.3 two types of incorrect response are appeared 14 students simply ignored decimal point and put- 0.3 at -3 , whereas some (34) students ignored negative sign and placed it at 0.3 . For 0.2 three types of incorrect responses has been observed. Some (87) students placed 0.2 at -0.2 and showed opposite sign misconception. Similar thinking has been shown by 34 students for -0.3 as in above case. 12 students ignore decimal part and place 0.2 at 2 , and 10 students placed it at -2 respectively. Here two types of misconceptions can be observed; ignore decimal point and opposite sign misconception.

This part of test (instrument) contains items placed on vertical number line.

\subsection{Placement of $(1,-2)$ negative and positive integers on vertical number line}

The analysis of students responses shows like on horizontal line they don't have any problem in locating integers on vertical line. Only some (14) students wrongly place -2 at 2 . They showed opposite sign misconception.

\subsection{Placement of $(-1.7,-2.1)$ negative decimals with nonzero integer part on vertical number line}

The students' incorrect responses for - 1.7, 189 students showed opposite sign misconception and placed it at 1.7. 20 students' responses revolved around zero. 10 has placed -1.7 at $-0.3(=-1+0.7)$ and 10 has placed at $0.3(=1-0.7)$. For $-2.1,88$ students ignored negative sign showing opposite sign misconception and placed -2.1 at 2.1. Some (4) students placed -2.1 at $-1.9(=-2+0.1)$ and 4 
at $1.9(=2-0.1)$. So here three types of different in correct consistent responses are found.

\subsection{Placement of $(2.3,1.8)$ positive decimals with nonzero integer part on vertical number line}

In both the distributions, students use similar thinking as they (35) place 2.3 at $1.7(=2-0.3)$ by translating negative interval to positive region and some (10) has placed it at -2.3 (opposite sign misconception). For 1.8 some (7) studnets placed it at $0.2(=1-0.8)$ and some placed it at -1.8 .

\subsection{Placement of $(-0.2,0.3)$ negative and positive decimals with zero integer} part on vertical number line

Students' responses for decimals with zero integer part are analyzed. 189 students placed -0.2 at 0.2 (opposite sign misconception). Remaining students placed it at -2 and 2 . They ignored decimal part and interpret it as whole number. For $0.3,53$ students incorrectly placed it at -0.3 (opposite sign misconception) and 4 students put at 3 and -3 (ignore decimal point).

\section{Discussion}

The careful analysis of students' incorrect responses on horizontal and vertical number line lead to four types of misconceptions. The consistency in their incorrect response pattern supports about the argument regarding systematic errors is misconception. Although the responses showed placing integers and decimals on vertical number line seems difficult for them as more students' responses are incorrect in this part of test, but the consistency between incorrect responses has been observed in both (horizontal and vertical) number lines.

\subsection{Opposite sign misconception}

The students' responses showed, they have ignored negative sign while placing -1 on horizontal and -2 on vertical number line, they wrongly placed it at 1 and 2 . The same thinking has been observed by their responses while placing 1.8 and -2.3 on horizontal and -1.7 and -1.2 on vertical number line they have ignored negative sign and place the decimals in positive region of number line. The positive decimals like 1.7 and 2.3 for horizontal number line have been placed in negative region of number line. In almost all responses for positive and negative decimals on horizontal and vertical number line such type of response pattern has been observed i.e. they placed the number with opposite sing. For the analysis of decimals with zero integer part the same type of response pattern has also been observed. They place -0.3 at 0.3 and 0.2 at -0.2 , instantly we can say they have used opposite sign misconception in consistent to their previous responses. 
Students' response simply showed students are unaware of negative and positive integers. Such responses of students were also found by Faudiah, Suryadi \& Turmudi (2017) they found 97\% students are unaware about the sign of integers. This shows students difficulty about dealing number line. The other possibility of wrong answers may be they use number line only started from 0 only positive side of number line. Here they found two types of problems one dealing with sign of integer and other is dealing with decimals. The third possibility may be negative numbers are abstract. The magnitude of negative numbers is also confusing for them such as -6 is less than -3 which is not true otherwise so the magnitude and direction of negative numbers is creating confusion for students.

\subsection{Translating positive interval misconception (TPI)}

Translating positive misconception was defined by Widjaja, Stacy and Steinle (2011). According to them the students with TPI misconception knows the location of numbers and integers on the number line. The position of negative decimals however are incorrectly founded by translating the intervals of positive decimals between integers to the negative region, such as interpreting -1.2 as ($1-0.2)$ they interpreted it as $(-1+0.2)$. This type of responses also been observed by the student in current study for placing negative decimals on horizontal and vertical number line. Such as for placing decimals on horizontal number line they interpreted -1.8 as $(-1+0.8)$ and placed it at -0.2 . Similar response has been observed for -2.3 they have interpreted it as $(-2+0.3)$ and placed it at -1.7 . For vertical number line similar behavior has been observed, they place -1.7 at $-0.3(=-1+0.7),-2.1$ has been placed at $-1.9(=-2+0.1)$.

These students know the location of negative integer part of decimal on number line but the placement of decimal part with integer is problem for them. They systematically translated the decimal part to positive side in negative region of number line. This misconception seems to have roots in above "opposite sign misconception" as they don't know the signs of integers and decimals so this problem occurs while locating decimals on number line. The knowledge of magnitude and direction of numbers seems the main reason for this misconception.

\subsection{Translating negative interval misconception (TNI)}

The students with TNI misconception showed similar responses for negative and positive decimals. For positive decimals like 2.1 and 1.7 on horizontal number line and 2.3 and 1.8 on vertical number line they are translating the intervals of negative numbers between integers to the positive region. Such as instead of interpreting 2.1 on horizontal number line as $(2+0.1)$ they interpreted 
as $(2-0.1)$ and placed at 1.9 , further they interpreted 1.7 as $(1-0.7)$ and placed at 0.3 . Similarly they interpreted 2.3 as $(2-0.3)$ and 1.8 as $(1-0.8)$ and wrongly placed at 1.7 and 0.2 respectively. Whereas for negative decimals they translated the interval of negative number between integer to the positive region on vertical number line only, such as instead of interpreting -1.7 as $(-1-0.7)$ they interpreted as $(1-0.7)$ and wrongly placed at 0.3 similar response has been observed for -2.1 .

This type of misconception has not been reported in literature not even by Widjaja and colleagues. This type of thinking may have roots with negative thinking was described by Steinle (2004, p. 476) and Steinle $(2004$, p. 56). She said students with negative thinking confused decimals with negative numbers. Previously, Steinle and Stacy (1998) interviewed a pre-service teacher about decimals $0.20>0.35,2.516>2.8325$ etc. the explanation given by respondent as: "I was thinking along a number line and considering decimal numbers to be equivalent to negative numbers. Therefore - 20 was larger than - 35". This was further elaborated as S behavior by Steinle (2004). The translating negative interval misconception could be an extension of such $\mathrm{S}$ behavior. The thinking about decimals are negative numbers may be influenced students such type of responses and they subtracted the decimal part from integer while placing decimals on number line. But the difficulty is with the responses of negative decimals as while interpreting -1.7 as $(1-0.7)$ the integer part has been considered as positive and decimal part has been subtracted in continuation of decimals as negative numbers. It needs further clarification.

\subsection{Decimal point ignored misconception}

This misconception has been identified by students' responses when they incorrectly placed decimals with zero and non-zero integer part. These students incorrectly placed 0.2 at 2 and -2 on both sides of the number line. Similarly 0.2 placed at 2 and -2 and 0.3 at 3 and -3 . Decimal point ignored thinking as misconception was first noticed by Swan (1983). The students with this type of thinking ignored the decimal point and treat decimals as a whole number like 1.34 as 134. It was further elaborated by Steinle (2004) as "L" behavior. The situation explained there, is not similar to the situation here but the responses showed similar thinking behind it, while placing decimals on number line they ignore the decimal point and place decimal as a whole number on number line.

The thinking of such students was also described by Stacey (2005) as L1 way of thinking. In the L1 thinking students interpreted decimals part as whole number ( $p, 24)$. They explained it as 4.63 is $>4.8$ because 63 parts are more than 8 parts they ignored the decimal point. The above response is the extension of L1 behavior in the way when student tried to place decimals with zero integer part 
they ignore decimal point and they interpreted the decimal part as whole and place 0.2 at 2 . They have also placed 0.2 at -2 this is something like opposite sign misconception. This is an example of how student's knowledge grows by adding or overlapping some new and previous understanding or, misunderstanding of concepts. This is extension in their previous concept by using the same they have interpreted the decimal part as whole number along with it they have placed these numbers on inverse region of number line.

\section{Conclusions}

The discussion above has explained students' thinking regarding placing integers and decimals on horizontal and vertical number line. The analysis of students' incorrect responses leads to four misconceptions. These misconceptions are not new previously it has been observed by students' responses in one or the other way while dealing with decimals and integers. The description of these misconceptions rooted in previously existed misconceptions. Students have opposite sign misconception as they place negative and positive integers in alternative region of number line. Further, they have TPI and TNI misconceptions as they focused integer part as whole number and translated interval of decimal part towards positive and negative region of number line. The fourth they have decimal point ignored misconception and they considered zero (of decimals with zero integer part) as disregarded part, as adding zero before number does not change the number, similarly when decimal point has been ignored the left is whole number which seems easy to place on number line. It is felt that students' ways of thinking may be further elaborated by some sort of interviews to find out the reason behind such type of thinking.

\section{Recommendations}

It is found that students' misconceptions have some roots in their previous learning. These misconceptions need to be addressed at the conceptual development stage. The visual description may be helpful in eliminating confusions based on abstract conceptual understanding. It is further suggested that the gap between conceptions and misconceptions regarding integers and decimals should be adequately addressed by teachers at the initial stage through specialize instructive medium. It is further recommended that concept clarity may be focused. The knowledge about the magnitude of numbers and direction of integers and decimals (positive and negative) on number line may be considered by teachers during teaching. Special exercises and practice is recommended for the clarification of students before advancement to next concept. FDE needs to design training for teachers as the teachers may be the root cause of such misconceptions transferring to students. 


\section{References}

Diezmann, C. M. \& Lowrie, T. (2006). Primary students' knowledge of and errors on number lines. In Grootenboer, P. and Zevenbergen, R. and Chinnapan, M. (Eds.). Proceedings $29^{\text {th }}$ Annual Conference of the Mathematics Education Research Group of Australasia, (pp. 171-178). Canberra.

Fuadiah, N. F., Suryadi, D. \& Turmudi. (2017). Some difficulties in understanding negative numbers faced by students; A qualitative study applied at secondary schools in Indonesia. International Education Studies, 10(1), 24-38.

Hannula, M. S. (2003). Locating fractions on a number line. In N.A. Pateman, B. J. Dougherty, \& J. T. Zilliox (Eds.), Proceedings of the $27^{\text {th }}$ conference of the International Group for the Psychology of Mathematics Education (Vol. 3, pp. 17-24). Honolulu, HI: Psychology of Mathematics Education.

Irwin, K. (1995). Students' images of decimal fractions. In L. Meira, \& D. Carraher (Eds.), Proceedings of the $19^{\text {th }}$ conference of the International Group for the Psychology of Mathematics Education (pp. 50-59). Reclife, Brazil: PME.

Mackinlay, J. (1999). Automating the design of graphical presentations of relational information. In S. K. Card, J. D. Mackinlay, \& B. Schneiderman (Eds.), Readings in information visualization: Using vision to think, (pp. 66-81). San Francisco, CA: Morgan Kaufmann.

Mclntosh, J., Stacey, K., Tromp, C., \& Lightfoot, D. (2000). Designing constructivist computer games for teaching about decimal numbers. In J. Bana, \& A. Chapman (Eds.), Mathematics education beyond 2000. Proceedings of the 23rd annual conference of the Mathematics Education Research Group of Australasia (pp. 409-416). Fremantle, Western Australia: MERGA.

Michaelidou, N., Gagatsis, A., \& Pitta-Pantazi, D. (2004). The number line as a representation of decimal numbers: A research with sixth grade students. In M. J. Hoines, \& A. B. Fuglestad (Eds.), Proceedings of the $28^{\text {th }}$ 
Conference of the International Group for the Psychology of Mathematics Education, (pp. 305-312). Bergen, Norway: PME.

Peled, I., \& Carraher, D. (2008). Signed numbers and algebraic thinking. In J. J. Kaput, D. W. Carraher, \& M. L. Blanton (Eds.), Algebra in the early grades, (pp. 303-328). New York: NCTM.

Putt, I. J. (1995). Pre-service teacher ordering of decimal numbers: When more is smaller and less is larger!. Focus on Learning Problems in Mathematics, $17(3), 1-15$.

Schindler, M. \& Hußmann, S. (2013). About students' individual concepts of negative integer- in terms of the order relation. Proceedings of the Eighth Conference of the European Society for Research in Mathematics Education (CERME). Working Group 2, 373-382.

Schindler, M., Hußmann, S., Nilsson, P., \& Bakker, A. (2017). Sixth-grade students' reasoning on the order relation of integers as influenced by prior experience: an inferentialist analysis. Mathematics Education Research Journal, 29, 471-492.

Stacy, K. (2005). Travelling the road to expertise: A longitudinal study of learning. In Chick, H. L. \& Vincent, J. L. (Eds.). Proceedings of the $29^{\text {th }}$ Conference of the International Group for the Psychology of Mathematics Education (pp. 19-36). Melbourne: PME.

Stacey, K., Helme, S., Steinle, V., Baturo, A., Irwin, K., \& Bana, J. (2001). Preservice teachers' knowledge of difficulties in decimal numeration. Journal of Mathematics Teacher Education, 4(3), 205-225.

Steinle, V. (2004). Changes with age in students' misconceptions of decimal numbers ( $\mathrm{PhD}$ thesis, University of Melbourne, Melbourne, Australia). Retrieved from http://repository.unimelb.edu.au/10187/686.

Steinle, V., \& Stacey, K. (1998). The incidence of misconceptions of decimal notation amongst students in Grades 5 to 10. In C. Kanes, M. Goos, \& E. Warren (Eds.), Teaching Mathematics in New Times. Proceedings of the 21st annual conference of the Mathematics Education Research Group of Australasia (pp. 548-555). Gold Coast, Australia: MERGA. 
Steinle, V., \& Stacey, K. (2004). Persistence of decimal misconceptions and readiness to move to expertise. In M. J. Hoines, \& A. B. Fuglestad (Eds.), Proceedings of the $28^{\text {th }}$ Conference of the International Group for the Psychology of Mathematics Education (pp. 225-232). Bergen, Norway: Bergen University College.

Swan, M. (1983). Teaching Decimal Place Value: A Comparative Study of "Conflict" and "Positive Only" Approaches. Nottingham: Shell Centre for Mathematical Education.

Widjaja, W., Stacy, K. and Steinle, V. (2011). Locating negative decimals on the number line: Insights in to the thinking of pre-service primary teachers. Journal of Mathematical Behavior, 30, 80-91. 